\title{
Initiatives in crystallographic education
}

\section{Gervais Chapuis}

To cite this article: Gervais Chapuis (2011) Initiatives in crystallographic education, Crystallography Reviews, 17:3, 187-204, DOI: 10.1080/0889311X.2011.561840

To link to this article: https://doi.org/10.1080/0889311X.2011.561840

$$
\text { 冓 Published online: } 26 \text { Apr } 2011 .
$$

Submit your article to this journal ¿

$$
\text { Џ Article views: } 244
$$

Q View related articles $₫$

4 Citing articles: 2 View citing articles 준 


\title{
Initiatives in crystallographic education
}

\author{
Gervais Chapuis* \\ École Polytechnique Fédérale de Lausanne, BSP, 1015 Lausanne, Switzerland \\ (Received 11 January 2011; final version received 7 February 2011)
}

\begin{abstract}
The rapid development of the Internet has created new opportunities for teaching in general and it is our aim to show how the current evolution can best be exploited for crystallography education in particular. Currently, we can find a very large selection of media dedicated to crystallographic education extending from plain text and videos to the most elaborate forms of interactive simulations. However, due to the lack of criteria for ranking educational materials, it is difficult to select the most appropriate information for educational purposes. In this article, we propose a modular approach to the teaching of crystallography along the lines of the Kahn Academy in the field of mathematics. Each module can be an interactive application (applet), a short video or any other form of media which can be used by teachers and learners to illustrate the fundamental concepts of crystallography. This includes, among others, topics on the symmetry of periodic objects, diffraction phenomena, the Fourier methods of density calculations and the representation of structures depending on the physical or chemical phenomena that one would like to stress. The modular approach resides in the freedom given to the teachers in selecting the topics which are best suited according to their visions, needs and pedagogical aims.
\end{abstract}

Keywords: crystallography teaching; web-based teaching; crystallography applets; e-science

\section{Contents}

\section{Introduction}

2. Current status in teaching activities

3. Choice of resources available on the web

4. Examples of useful educational resources

4.1. Crystal symmetry

4.2. Diffraction

4.3. Fourier transform

4.4. The charge flipping algorithm for the resolution of the phase problem

4.5. Structure representation

\footnotetext{
*Email: gervais.chapuis@epfl.ch 
6. Conclusion and future vision 201

Notes on contributor 202

References $\quad 202$

Subject Index $\quad 203$

\section{Introduction}

Crystallography is typically a field of science at the intersection of many fundamental branches including chemistry, physics, life and environmental sciences. This position is certainly an advantage but it is also the origin of an identity problem which is well known among crystallographers involved in teaching their speciality. At least in Europe, the tendency to limit the number of hours in the bachelor and master curricula has affected the place of crystallography teaching. Only in a few faculties is crystallography, still taught at the bachelor level. At the master level, the situation is not particularly favourable either. Moreover, crystallography courses are seldom mandatory and most of the time, they are taught as options. This trend in teaching crystallography at the academic level has direct consequences on the way it is taught and thus requires some new thoughts in order to adapt and optimize the educational methods for this branch.

Traditionally, and due to the specific nature of their speciality, crystallographers have always been early adopters of the most advanced computational resources, either for the determination of crystalline structures, for the optimization of structure models by leastsquares refinements, for the calculation of three-dimensional (3D) Fourier synthesis or for storing large amounts of information in databases. For example, crystallographers have been keen to take advantage of the new possibilities currently available on the Internet, where we observe the trend of disentangling hardware and software resources. In other words, more and more applications become platform independent and can thus be directly accessed from a browser. The cpu power of the current laptops and the development of efficient algorithms has improved to such an extent that even Fourier transforms (FTs) or optimization procedures can be done in an instant.

The direct consequence is that many useful applications can be easily accessed, in particular for teaching crystallography. Numerous types of dynamic simulations illustrating different aspects of crystallography e.g. structure representation or diffraction methods can be directly obtained from a few clicks on a browser. For educational purposes, not only professional crystallographers can exploit this excellent opportunity in seminars or lectures for demonstration purposes, but also the students can improve their knowledge by using the tools which are at their disposal on the web.

In this article, we will show what type of tools are freely available on the Internet and how they can be applied in order to facilitate the education in various fields of crystallography and thus palliate the shortcomings of the offers at the bachelor and even master levels. Let us look first at the current status of the teaching activities observed in the field of crystallography.

\section{Current status in teaching activities}

In this context, crystallography teaching will not be limited to any specific requirement of the student curriculum. We are concerned here with the basic principles of structural 
architectures, symmetry and diffraction which are sufficiently general to concern all students, independent of their orientation.

In some educational institutions, crystallography is taught in the classical way, i.e. lecture, exercises and experimental work. Unfortunately, we observe a tendency to restrict the contact of students with crystallography to a very limited number of practical sessions without any proper introduction to the field and consequently without any theoretical background. Another common worry is how we might reduce crystallography teaching to learn how to use a structure solving package in front of a computer screen.

Most of the time, a textbook or some lecture notes are recommended for ex-cathedra courses. For some students, the acquisition of a textbook is too expensive and their first reaction is to find the equivalent on the Internet. For example, the International Union of Crystallography (IUCr) (http://www.iucr.org) provides a large collection of freely available pamphlets, short fascicules dedicated to specific subjects on crystallography. Some fascicules are up-to-date, e.g. those dedicated to symmetry aspects of crystal structures. This is unfortunately not the case for other subjects dealing with experimental techniques where new developments have rapidly changed the way they are taught. This concerns among others new detectors, new focalizing optics and new X-ray sources. Another problem to be mentioned is that the material prepared by some authors and downloaded from a website might not always fit the aim of the course created by the original author. This can sometimes cause difficulties for students unaware of the problem.

The limited offer of crystallography lectures at the bachelor and master levels has prompted a series of initiatives from the part of national academies or other organizations interested in training new students in the field. In the United States, the Cold Spring Harbor school (http://meetings.cshl.edu/index.html) and in Europe, the school organized by the British Crystallographic Association (http://www.dur.ac.uk/durham.X-ray-school/) are well established and periodically train new students. In the meantime, other schools have been established on a periodical basis as for example the Zurich school of crystallography (http://www.oci.uzh.ch/group.pages/linden/zsc/). The Cold Spring Harbor and Zurich schools distinguish themselves in that a large portion of the time is dedicated to hands-on sessions where students are supposed to solve structural problems. In the Zurich school, students can even bring their own crystals and solve their structures to bring home at the end of school. Many more schools are organized for example as satellite meetings of important international conferences. They are unfortunately not repeated periodically and thus it is difficult to evaluate their usefulness.

Recently, and probably due to the steadily decreasing offer of crystallography courses in higher education curricula, a tendency to establish doctoral schools of crystallography at the national level has been observed, as is the case for example in Spain.

In this context, we observe a rapid evolution of the teaching programs and methods, not only in crystallography but also in other branches of science. The purpose of this article is to show how we can take advantage of the new technologies currently at our disposal on the web and freely available in most cases.

\section{Choice of resources available on the web}

The IUCr website (http://www.iucr.org/) lists a series of educational resources available on the web for the purpose of crystallography teaching. The site provides various links to resources ranging from pure text files to videos and interactive simulations. It also includes 
pointers for downloading software resources on your own computer. We have to note however that in some instances the proposed resources are not available anymore or are out-of-date. This is sometimes the case for web resources developed thanks to temporary grants. Their developers have probably long since moved to other activities, taking away with them the know-how and the software that created the resources. Fortunately however, there are still a good number of resources which are well maintained; some of them will be described below.

The private sector has also contributed to interesting educational programs. The Apple company for example is strongly promoting its iTunes $U$ site (http://www.apple.com/ education/itunes-u/) where a large number of educational institutions are publishing resources including slideshows, pdf files, videos and audiobooks. Currently (end of 2010) more than 350,000 are listed. But the current offer for crystallography is rather limited and contains essentially two ex-cathedra lectures in English, one given at MIT during a semester and the other dedicating a few hours to diffraction at Yale. Both lectures are video recordings of the teacher lecturing in front of their classroom. The most popular source of videos on the web, YouTube (http://www.youtube.com/), also contains a series of videos dedicated to the subjects of crystallography and diffraction. The didactical value of their contents is somewhat limited and the current search engines do not yet allow a selection according to didactical criteria or according to the difficulty level of the presentation.

An initiative worth mentioning is the use of mobile phones to develop pedagogical tools. The decoration of periodic patterns allowed by the 17 plane groups have been duly exploited by their developers and are already available on the dedicated websites where they can be downloaded $(1,2)$. The current processors are powerful enough to allow fast FT. It is to be expected that very soon we will be able to calculate electron densities for two-dimensional (2D) crystals or simulate diffraction patterns.

Perhaps the most important developments for crystallography on the web concern the so-called applets, i.e. small portions of software which are autonomous and platform independent and which can be downloaded directly from a browser and executed locally. Each applet can illustrate or simulate a specific concept which is usually taught in a crystallography course. Many existing applets can be used e.g. to simulate various diffraction methods and patterns or illustrate point and space group symmetry. One of the advantages of the applets is that the user can test various cases along a 'what-if' scheme. Another of their important advantages is to allow the teacher to use them during his course and to present the selection that best illustrates specific points. A complete course in crystallography might thus be built from a selection of applets developed by various authors depending on the requirements of the teacher and the offer which can be found on the web. On the other hand, the student can also make use of the same applets for a better understanding of concepts which may have been brought too quickly during the presentation of the teacher.

Applets are by no means the only possibility to simulate processes directly on the web. The use of computer servers is often favoured by some developers. In this model, the user sends the required data and receives the results almost immediately by means of his/her browser. This obviously requires a fast internet connection between the user and the server and is sometimes of advantage when the simulation is cpu intensive.

In the next section, we shall illustrate some of the simulations and applets currently available on the net, which can be used in real time to illustrate various aspects of an introductory course in crystallography. 


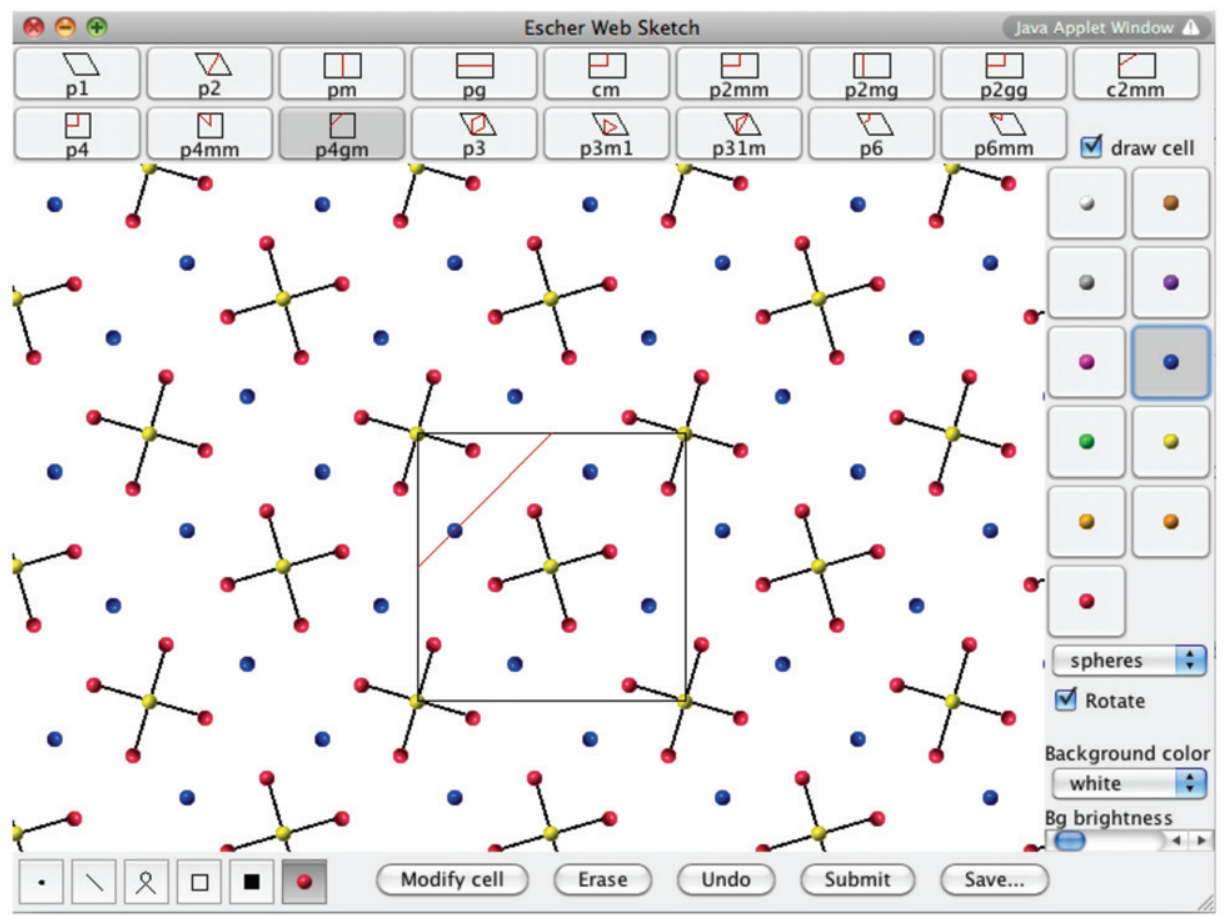

Figure 1. The Escher web sketch panel representing a hypothetical 2D structure with plane group symmetry $p 4 \mathrm{gm}$. The concept of general and special positions can be easily illustrated with this applet.

\section{Examples of useful educational resources}

A basic course in crystallography usually contains various topics including symmetry, diffraction and some crystal chemical considerations relating structures and physical properties. Diffraction phenomena are so closely related to FT that this topic must be included in a basic course of crystallography. We shall present different applets and simulations which can be directly used by the teacher to illustrate the different components of a crystallography lecture.

\subsection{Crystal symmetry}

The theory of symmetry in crystals is one of the most important components of a crystallography course. Notions like spatial periodicity, proper and improper rotation, glide planes and screw rotations are all symmetry components of the space groups. The topic of symmetry in crystals can easily be presented by considering first the 2D planar groups. Fortunately their number (17) is very limited as compared to the 3D space groups which amount to 230 . With the planar space groups, most symmetry operations can be illustrated with the exceptions of screw axis and roto-inversions. Escher Web Sketch (EWS) (3) is a very useful applet for the representation of planar groups (Figure 1). 
This applet can easily simulate any 2D structure, for example using a ball and sticks model structure. The concepts of repeating unit (unit cell) and symmetry operations with and without fixpoint can be illustrated by displacing an object on the screen and finding out if some of them are merging together at specific locations. Depending on the selection of the planar groups, we can observe that some of them do not exhibit any fixpoint, i.e. those consisting of translations and glide lines only. The concept of general and special positions is directly related to the notion of fixpoint operations, which is again related to the stoichiometric composition of chemical elements. Special positions have the property that atoms (balls) located on them generate only an integral fraction of the maximum number of symmetry equivalent positions which are generated by the symmetry operations of the planar group in a unit cell. The chemical composition given by the stoichiometry can be accommodated by selecting the adequate special positions satisfying the correct chemical formula.

As an exercise, the applet can for example be used to deduce all the possible special positions of a planar group and perhaps even determine the site symmetry group (point group) of the special position. The user can immediately check his findings by comparing his results with e.g. the Bilbao crystallographic server (http://www.cryst.ehu.es/) dedicated specifically to the symmetry theory of crystalline structures.

The EWS applet offers not only the possibility to represent crystalline structures in a plane but can also be used to create any possible $2 \mathrm{D}$ periodic decoration of the plane, like the famous arabic decorations often observed in mosques, or any other type of decoration with the set of tools offered on the control pane. The close relationship with crystallography and art can also be exploited with recent applications which have been published for mobile phones including the iPhone $(2,4)$. An interesting possibility of innovation for the developer is the gravity sensor and the accelerometer which are increasingly becoming part of the mobile hardware. This has been fully exploited for EWS adapted to the iPhone where new patterns can be stochastically generated by shaking the device with a given planar group symmetry.

The special positions of planar or space groups are characterized by their own site symmetry groups of which there are 10 in $2 \mathrm{D}$ and 32 in $3 \mathrm{D}$. The symmetry of the 32 crystallographic point groups or also called crystal classes is best represented by polyhedra. In this respect, a very useful applet called Polyhedra of Point Groups has been designed by Steffen Weber (5) which is illustrated in Figure 2. The user can select one of the crystal classes classified according to its crystal system and place a point anywhere on the stereographic projection. Different shapes of the polyhedron with the same symmetry can be generated by varying the position of the point on the stereographic projection.

Symmetry operations with improper rotations characterized by the international symbol $\bar{n}$ are often difficult to assimilate by the beginner. The applet Introduction to the symmetry of polyhedra (6) is very helpful to understand improper rotations by combining inversion and rotation. With a real object, the inversion operation is physically not possible without creating a new object. On a computer screen, however, the inversion can be easily done by migrating each vertex of the figure towards its inverse by crossing the inversion centre. The new figure is then rotated until it matches the original one. From the same applet, the symmetry operations of various polyhedra can be studied offering to the student the possibility to dynamically discover unexpected symmetry operations (typically two-fold axes normal to $\overline{4}$ in $\overline{4} 2 \mathrm{~m}$ ) along various directions of the polyhedra. 

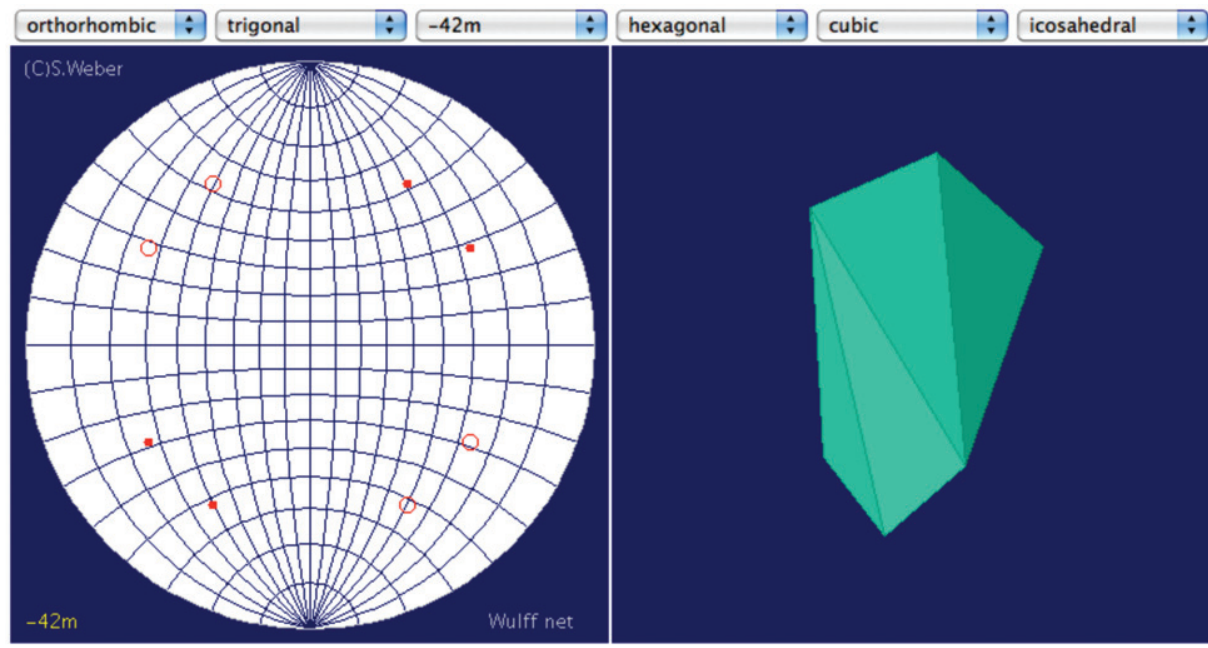

faces: 8 vertices: 6

Figure 2. Stereographic projection of the face normals and the corresponding polyhedron with point symmetry $\overline{4} 2 \mathrm{~m}$. Open circles and dots distinguish between face normals pointing below and above the projection plane.

\subsection{Diffraction}

The diffraction phenomenon is so central to crystallography that the two terms are often confused in some literature, in particular when the phenomenon is related to X-rays. A large number of presentations dealing with diffraction can be accessed directly on the web. They extend from written documents to dynamic simulations including videorecordings. Each presentation reflects, however, the teaching objectives of their authors and it is not surprising to find a large spectrum of complexity. In the present context, diffraction shall be approached by using the Ewald sphere and the reciprocal lattice. The advantage of this approach is that it can be used to simulate any type of diffraction phenomena provided that the object giving rise to diffraction is an object with space periodicity.

The interactive applet The reciprocal lattice calculator (7) leads the student step by step to obtain the reciprocal lattice derived from a $2 \mathrm{D}$ periodic pattern. The applet is able to first detect the two lattice vectors and consequently obtain the lattice nodes once an origin has been selected. From the study of different series of lattice lines, the student can generate a new series of points by taking normals to various series of lattice lines and reporting the inverse of the distance between the lattice lines and its multiples. The student can continue the exercise until he/she realizes that all the points generated by this method also form a lattice, the reciprocal lattice.

Once the reciprocal lattice is introduced, it is easy to simulate any diffraction pattern with the applet DiffractOgram (8) which is based on the Ewald sphere, the radius of which is given by the inverse of the wavelength of the incident beam which is also identical to the wavelength of the diffracted beam. In this model, diffraction takes place any time a reciprocal lattice point crosses the sphere. The diffracted beams generated from different 


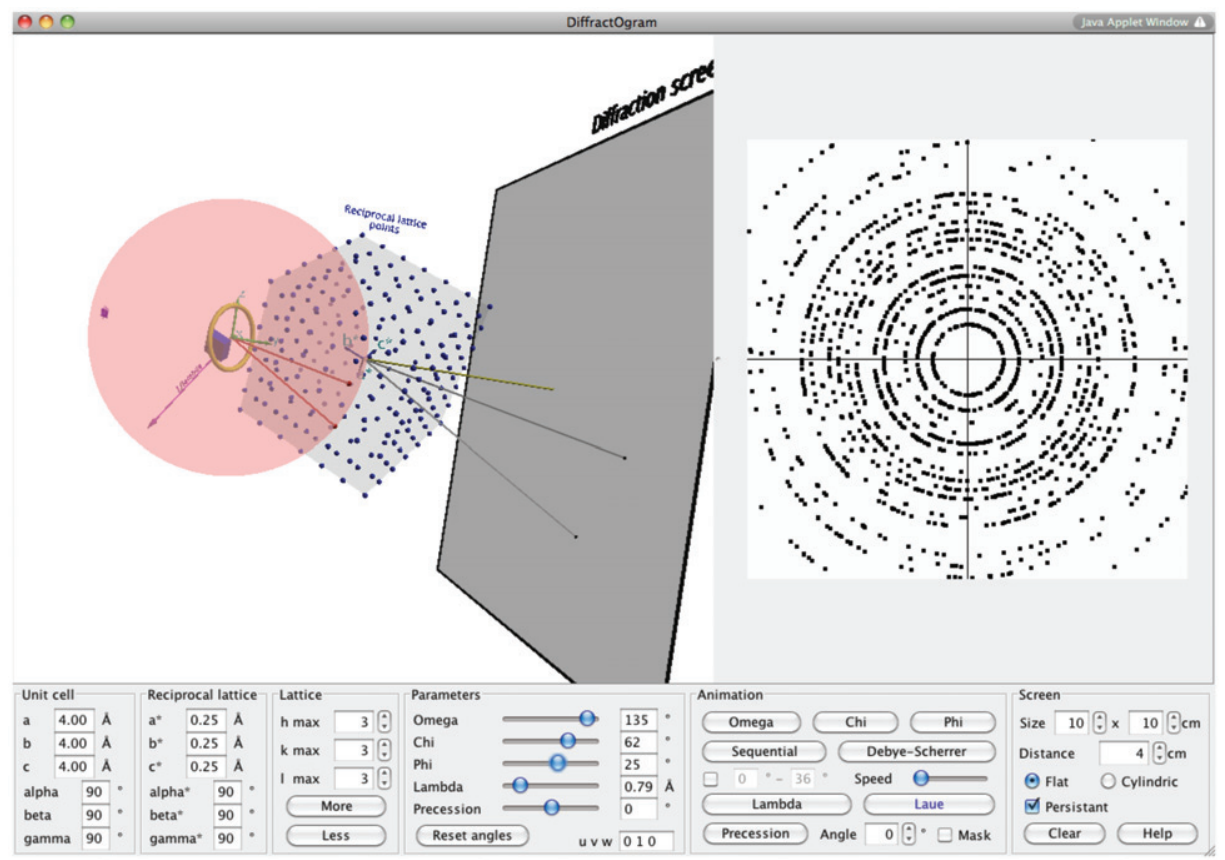

Figure 3. Applet panel for the simulation of different methods of diffraction. The simulation is based on the Ewald sphere, the crystal sample represented by its reciprocal lattice and the diffraction screen. The right screen illustrates a powder diffraction diagram on a flat screen.

orientations of the reciprocal lattice, i.e. from different orientations of the crystal, can be recorded on the diffraction screen, either a photographic film or nowadays an area detector.

The panel of the DiffractOgram applet is illustrated in Figure 3. The left pane illustrates the position of the crystal at the centre of a goniometer, the Ewald sphere and the diffracted beam reaching the screen for a particular orientation. The right pane represents the $2 \mathrm{D}$ detector accumulating all the impacts of the diffracted beams. The bottom pane contains all the control buttons arranged in different boxes of functions, allowing thus the simulation of a great variety of diffraction methods including Laue transmission and reflection, rotating crystal and precession. Powder diagrams can easily be simulated by sequentially orienting stochastically the crystal in all possible directions by means of the $\omega, \chi$ and $\phi$ angles of the goniometer. The right pane of Figure 3 specifically illustrates such a case after accumulating a large number of orientations. It is even possible to select between a flat detector or a cylindric one as in the classical Debye-Scherrer film method. In the Laue method, the diffraction screen can be placed both at positive and negative distances from the origin of the reciprocal lattice, thus allowing simulation of Laue diagrams in transmission and reflection modes. Contrary to the rotating crystal method, the precession method produces undeformed reciprocal layers provided that a diffraction screen is inserted. Although precession cameras have disappeared from the crystallography laboratories, the pedagogical benefit of this simulation is considerable for the student and worth the effort! 


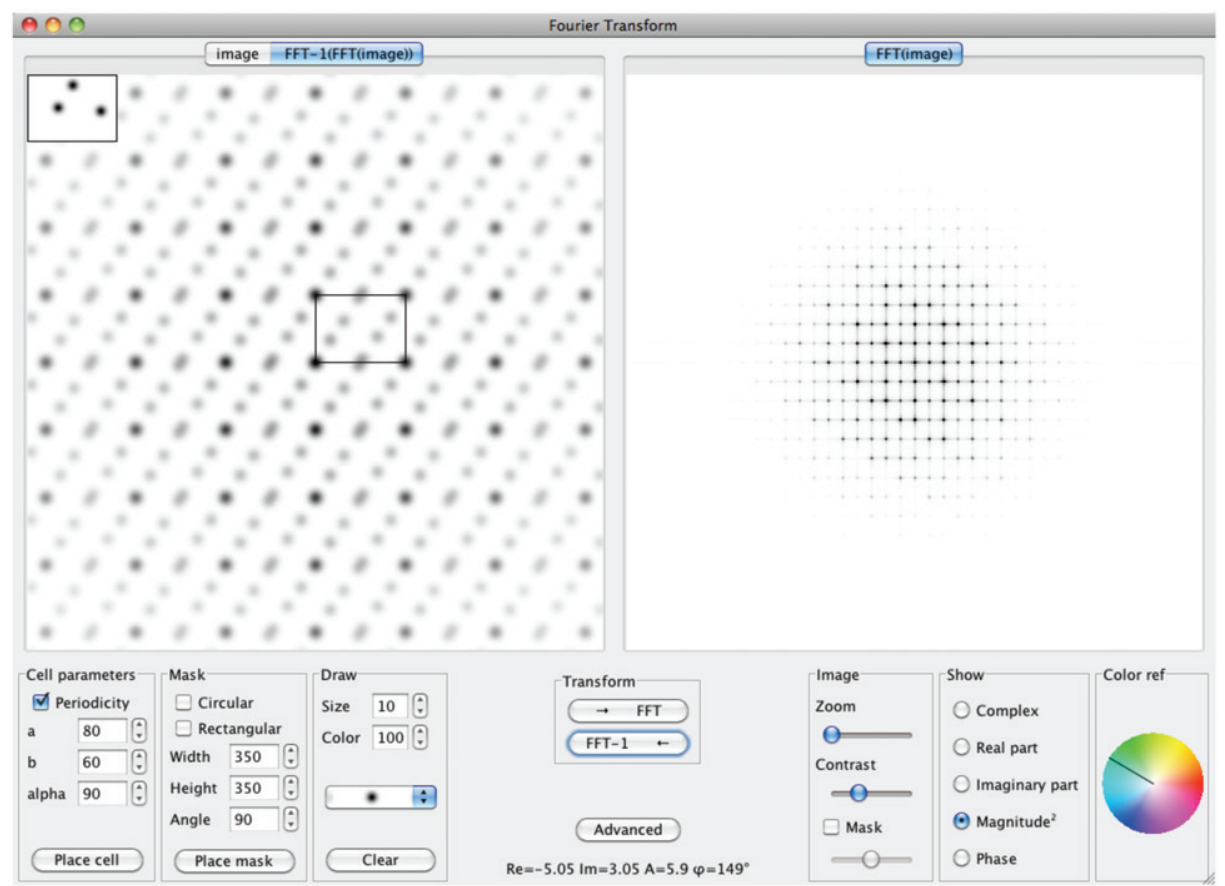

Figure 4. Applet panel for the calculation of Fourier and inverse FTs of $2 \mathrm{D}$ single or periodic objects. In this example, the left pane illustrates the Patterson function of the original structure given in the inset.

\subsection{Fourier transform}

The concept of FT is so central to the phenomenon of diffraction that it is most often treated as an integral part of a basic course in crystallography. The development of very fast algorithms for FT and the high frequency of the current cpu installed on each personal computer allows one to simulate in real time the diffraction pattern of any complex object including periodic ones, or inversely to obtain the electron density of a crystal structure provided that the phases and amplitudes of the coefficient of the inverse FT are known. The applet Fourier transform (9) fulfills exactly this role and represents a great step forward in comparison to the atlases of FT which were popular among crystallographers before fast cpu and algorithms were available.

The panel of the applet Fourier transform is represented in Figure 4, again with a left and right pane, both of them controlled by the bottom pane. The tools on the lower left are used to generate any object, periodic or not. The Fast Fourier transform (FFT) button generates on the right pane the FT of the left figure. Inversely, the button $\mathrm{FFT}^{-1}$ generates on the left pane the inverse FT of the right figure.

This figure also illustrates how to generate the Patterson function of a periodic structure. The rectangular box on the top left of the left panel is the original (periodic) structure which gives rise to its FT on the right. This picture is again transformed by inverse FT and the result is given on the left pane. One wonders why any picture first transformed and then inverse transformed is not equal to the original one? In the present case, we have used one of the convenient possibilities offered by the applet. It is indeed 
possible to play with the amplitude and phases before generating the inverse FT of the image. For example, the 'Show' pane allows one to play with the phases and amplitudes of the Fourier coefficients. The 'Magnitude' ${ }^{2}$ button substitutes all the phases by zero and replaces all the amplitudes by their squares. This is exactly the way the Patterson function of a periodic structure is generated. This option has been selected and therefore the figure on the left represents the corresponding Patterson function of the original structure.

The possibilities offered by the applet Fourier transform are numerous and greatly help the student to get a better understanding of the theory of FT. The left pane contains a series of practical tools to create periodic (e.g. 2D crystal structures) as well as nonperiodic objects (e.g. a single molecule). This can be useful to understand the principles of coherent scattering which are gaining importance with the development of new powerful $\mathrm{X}$-ray sources. The use of masks is also very useful in order to illustrate the effect on diffraction of a limited number of repeated units. Two types of masks can be selected, either rectangular or circular, as well as a selection of their dimensions. The masks can also be selected on a diffraction pattern, for example in order to illustrate the effect of a limited resolution on the inverse FT.

A series of advanced tools is also available to play with images that can be uploaded in the applet. The well-known illustration of the relative importance of phases and amplitudes in an FT process can be readily demonstrated with this applet. For example, it is possible to combine the phases of the FT of the portrait of a person A with the amplitudes of the FT of the portrait of a person B. The result of the inverse FT of the combined data readily gives the portrait of A thus clearly showing the importance of the phases over the amplitudes in FT. Experienced crystallographers are already familiar with this concept. Before the availability of automatic diffractometers, many structures could be solved by just estimating visually the intensities on a Weissenberg diagram as was done e.g. for the structure of yttrium trifluoride (10).

The role of each term in the summation of Fourier series can be nicely illustrated with another didactical applet developed by Kevin Cowtan (11) and represented in Figure 5. The panel is divided in four quadrants. In a first step, the $h k$ node of the reciprocal lattice is selected in the first quadrant. The amplitude and phase of the Fourier coefficient is then selected in the form of a complex number in the third quadrant and finally, the corresponding term is added to the Fourier summation by pressing the Set $S F$ button in the fourth quadrant. The resulting effect on the electron density function can be observed in the second quadrant. The user can easily observe the role of each Fourier term by selecting one of the reciprocal lattice points in the first quadrant. The effect of the phase selected in the third quadrant can be immediately observed on the second quadrant in the form of an undulating curve extending along the direction of the reciprocal lattice vector.

Another website dedicated to diffraction tutorials is worth mentioning. This site was developed by Thomas Proffen and Reinhard Neder and gives a step by step introduction to diffraction (12). The particularity of this site is that the simulations are done on a remote server maintained by the authors. The parameters are selected on the browser and then sent to the server for calculation. Almost instantly, the results are displayed on the browser. The number of possible types of simulations are numerous. The user is introduced successively to the concept of reciprocal space, to the convolution theorem and to the phase problem. The simulations also include some advanced topics like diffuse scattering resulting from modulated, disordered or defect structures. 


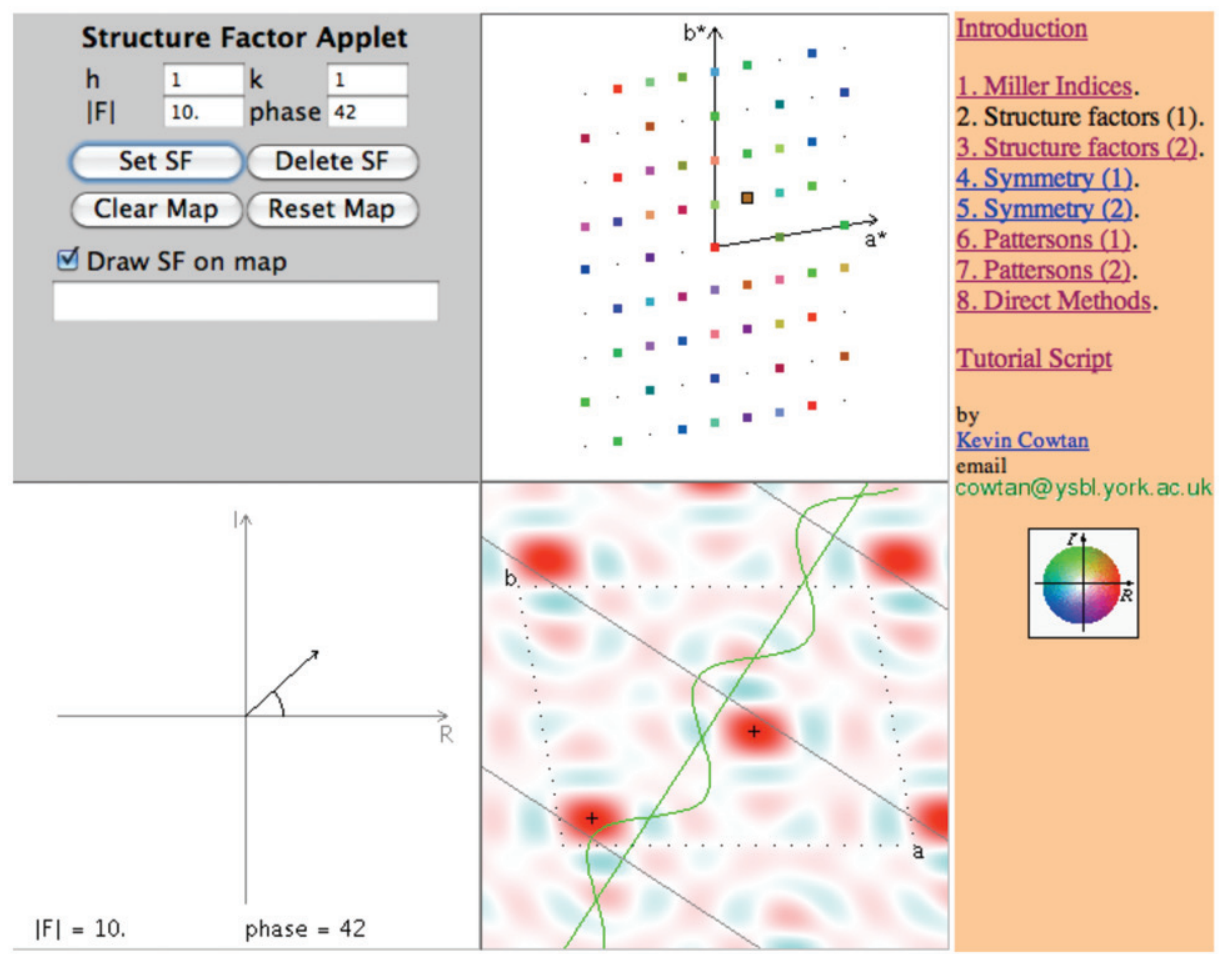

Figure 5. The structure factor applet illustrates the role of each term of the Fourier summation for calculating the electron density of a $2 \mathrm{D}$ structure.

\subsection{The charge flipping algorithm for the resolution of the phase problem}

The role of the FT in crystallography is particularly well illustrated in the recently discovered charge flipping $(C F)$ algorithm $(13,14)$. This very efficient phase recovering method is based on a cyclic iteration between direct and reciprocal space by using the FFT and inverse FFT algorithms. Here again, the applet charge flipping represented in Figure 6 can best illustrate the procedure by following in real time the various steps of evolution of the method.

The applet panel is divided in different panes. First, the middle part of the upper pane illustrates the various steps of the CF algorithm. The user can start with a step by step approach where each step is highlighted. Once the basic principle is understood, the user can continue the procedure, cycle by cycle or automatically, up to the final resolution of the structure. The CF applet starts by creating an arbitrary structure on the left pane with dedicated tools. This structure is then Fourier transformed to get the starting amplitudes, discarding the phases. It is the task of the CF algorithm to reconstruct the structure only on the basis of the structure amplitudes. In the present applet and for the first cycle, the phases are set randomly. For the subsequent cycles, the phases are calculated by the CF algorithm as indicated in the schematic representation of Figure 6. The horizontal pane of the applet records the evolution of the algorithm by plotting, among others, the value of the residual which should converge to a value similar to the reliability factor $R$ used in 


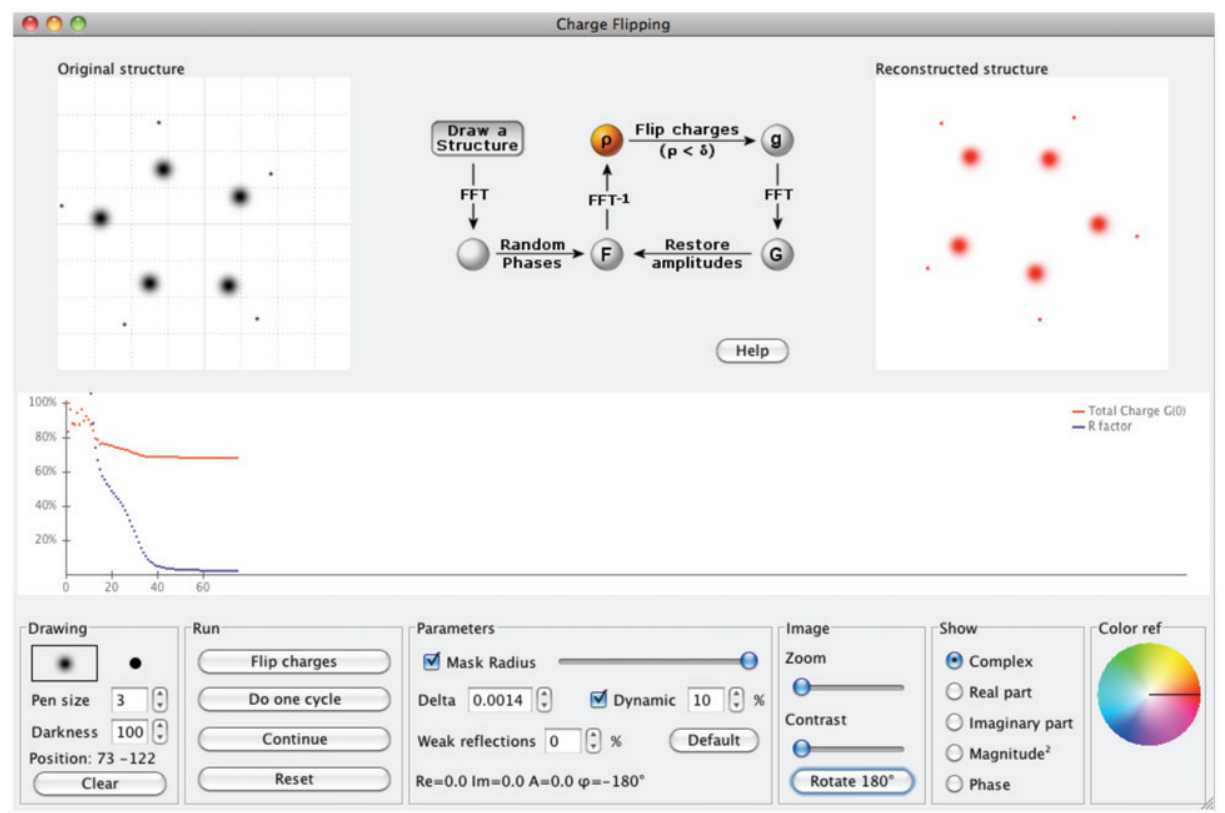

Figure 6. Control panel of the $\mathrm{CF}$ applet for the solution of the phase problem. The user can create an arbitrary structure and follow its resolution by the algorithm.

standard structure refinements. The procedure can be stopped once the structure is solved. One should mention here that due to the nature of the CF algorithm, the selection of the origin of the structure is arbitrary. Moreover, the solved structure can be rotated by $180^{\circ}$. Appropriate tools can be easily used to compensate for these features.

The CF algorithm requires only one single parameter $\delta$, a small positive value linked to the uncertainties of the data. In the applet, this parameter is estimated on the basis of the data. In some cases, however, it is necessary to modify this value in order to solve the structure. This can be done interactively in the control pane. By pressing the help button, the user can obtain the description of the method and the terms used and get some more insight into the resolution algorithm.

\subsection{Structure representation}

The last topic of interest for educational material which we shall present in this article concerns the representation of structures. The number of high quality softwares commercially available are numerous but unfortunately come at a price. Along the line of applets presented above, our aim is to represent any structure of reasonable size with a few clicks on a browser. This is precisely what the applet CrystalOgraph (15) allows one to do.

In order to describe the functionalities of the applet, the easiest approach is to select a structure from a database. The applet is able to read any CIF file, i.e. a standard description file containing among others all the data required to represent a crystal structure. The applet is also able to search the ICSD database (16) according to the 


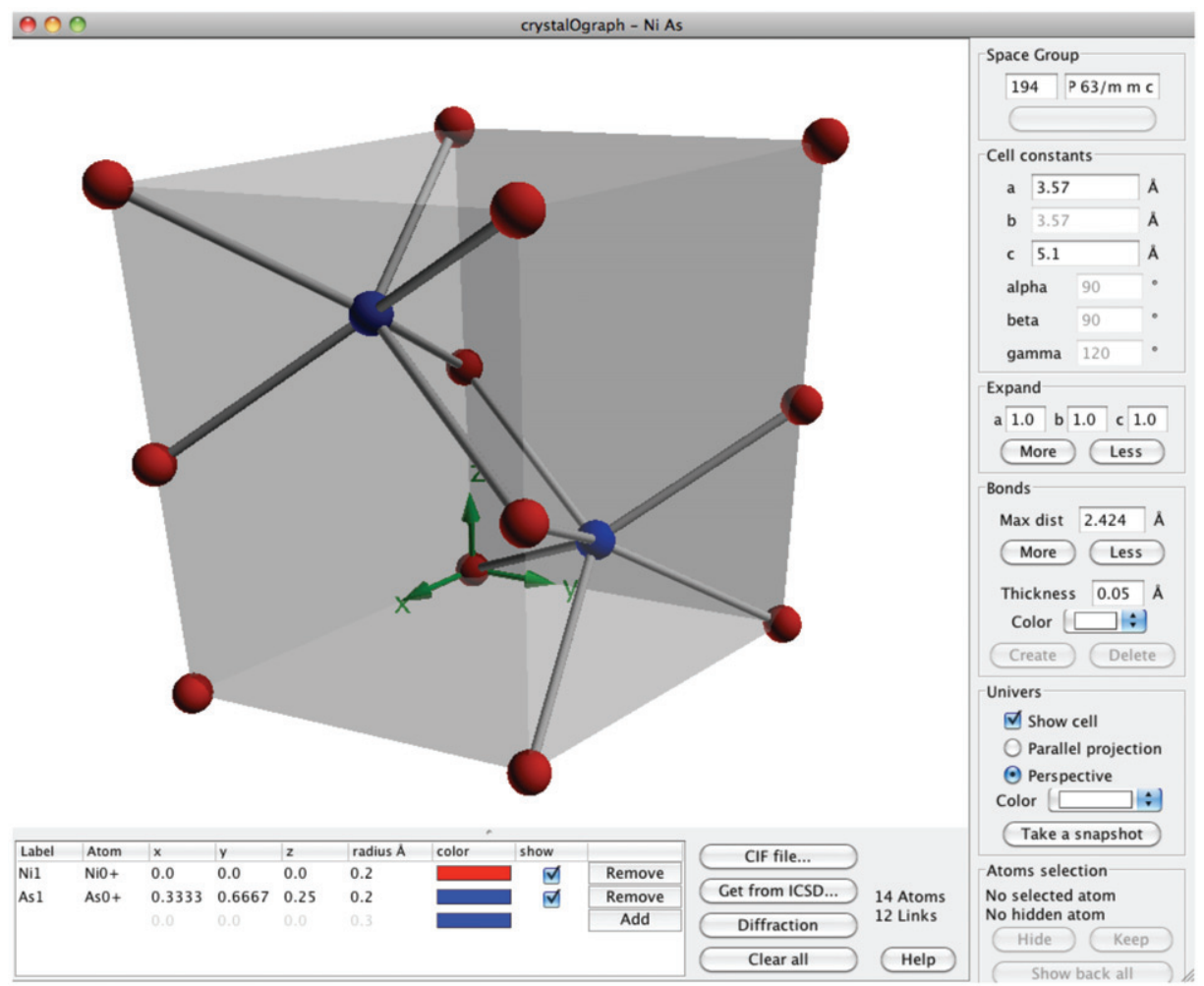

Figure 7. Representation of the unit cell content of NiAs. Space group symmetry, lattice constants and coordinates of the symmetry of independent atoms are listed in the left and bottom panes.

elements contained in the formula. We have to mention however that for licensing restrictions, only a limited number of possible hits can be extracted from the database. This restriction is fortunately of no consequence for educational purposes. Without any additional manipulations the structure is directly displayed on the screen with lattice constants, space group symmetry and atomic coordinates of the symmetry independent atoms, as illustrated in Figure 7. Structures can also be introduced by hand provided that the lattice constants, atomic space group symbol and atomic coordinates are introduced first. Any external CIF file can also be accepted as an input for the structure.

Various tools are at the user's disposal to obtain a deeper insight into the architecture of the structures. The applet can automatically and selectively find the interatomic bonds in terms of increasing distances. The content of the unit cell can be expanded at will and independently in the three space directions in order to analyse the structure in terms of layers, closely packed layers or any other useful criteria. Atomic sites can also be switched off in order to analyse partial structures. The display of the structures can also be controlled and orientations can be easily selected with the mouse.

It is also possible with the Diffraction button located on the control pane of the applet to simulate the diffraction pattern of the structure represented on the screen. The effect is to activate the reciprOgraph applet (17). The diffraction pattern can be either displayed as a 3D intensity weighted reciprocal lattice or as a powder diffraction diagram. The reciprocal 
lattice can be analysed in terms of reciprocal layers, thus giving the student the opportunity to analyse, e.g. the systematic absences depending on the space group symmetry.

\subsection{Exercises}

Every teacher knows perfectly well that any form of teaching requires some practical exercises where the learner can put into practice the concepts which have been acquired in the theoretical presentation. By nature crystallography requires a large amount of concepts to be understood in order to be familiar with the $3 \mathrm{D}$ aspects of atomic structures with their laws of symmetry and diffraction. An electronic course specifically developed for the Internet (18) includes some 50 exercises covering a broad spectrum of crystallographic notions belonging to a basic curriculum. Most exercises can be solved by using the applets presented above.

\section{Issues with Internet resources}

It should be clear at this point that the web offers a great potential for the future of learning. The educational resources available on the web cover almost all forms of media, from printed sheets of paper to the more elaborate forms of interacting applications and videos. The list will probably increase with the appearance of electronic books which can easily integrate all forms of educational material. One should nevertheless be aware that each new electronic device necessitates new software platforms which could probably be incompatible with older hardware.

Most of the developments of teaching materials are done by specialists of the field who are often responsible for the software maintenance of the applications they are developing. A great number of initiatives dedicated to the creation of educational materials have been developed thanks to the attribution, for a limited amount of time, of grants from national or international organizations. Once the grant is expired the maintenance of the application is seldomly secured for the future. If the application is only text based, its life cycle will most probably be longer than more elaborate forms using advanced software and hardware platforms. Several of these developments have already disappeared from the web due to the lack of financial support and consequently to the lack of specialists able to maintain them.

Copyright problems represent another issue of concern for web-based teaching material. It is not seldom that some applications are only available with special permissions granted only for students or members of a single educational institution. The only way to remove these restrictions is to appeal to developers and convince them to avoid using any proprietary materials for the development of their applications.

There are fortunately solutions to extend the life time of web-based applications and educational materials. This is where national and international scientific unions can play an important coordinating role. In the field of crystallography for example, the IUCr has already taken over the maintenance of a limited number of educational materials accessible through the web. We can hope that in the future this tendency will be further developed at an increased pace. Every effort should be made in order to convince executive committees of scientific unions to establish structures which are able to take over the maintenance of useful applications and consequently to slow down their normal tendency to disappear. 
Commercial organizations may also play an important role in maintaining educational materials available on the web. iTunes $U$ (http://www.apple.com/education/itunes-u/) and YouTube (http://www.youtube.com/) for example maintain a large number of video sequences and a few other selections of media. Here, I am convinced that any storage of information with these organizations is much more reliable than the one provided by some local server installed in a specific educational institution, where the responsibility of the maintenance usually depends on a single person.

\section{Conclusion and future vision}

The world of education is a very complex field and a science in itself. The teaching of crystallography is no exception. During their career, every teacher accumulates a series of teaching experiences and develops some optimal way based on a selection of textbooks, documents and publications. Only the unexperienced teacher would strictly follow the chapters of a dedicated textbook. Therefore, it is very unlikely that a full course of crystallography found on the web would suit the needs of experienced educators. Most probably the best contribution to teaching material from the web would be a complete series of modules, each one presenting a unique and limited topic of the field. Each module could have different forms, for example, a limited text, a small video or an applet.

The concept of the Khan Academy (19) in the field of mathematics is specifically based on this modular approach which explains the reason for its great success. This site lists more than 1600 videos recorded by Salman Khan and stored on YouTube. The subjects cover mathematics, basic sciences and finance. Each video explains a single topic and lasts from a few minutes to at most a quarter of an hour. Visitors of the Khan Academy can also test the acquired knowledge by accessing the pages dedicated to exercises.

In crystallography, the series of applets and other simulations which have been presented in this article are also based on the modular approach to web-based teaching materials. Each module can be used independently, either by the teacher or by the learner, for a better understanding of a specific topic or for training. This type of approach is also more compatible with the most recent trends in teaching where a more personal education, better adapted to the needs of each individual, is required. In this respect, I doubt that a sequence of video recordings showing complete series of lectures during a full semester would satisfy the criteria for good educational material. Here, the most important part of a lecture, namely the following interaction between the students and the teacher is missing. The use of modular materials is a useful way to circumvent this missing interaction.

One important positive aspect of web-based teaching materials should be mentioned here. This concerns the evolution of the material. New versions can be prepared and uploaded on the web at any time by the developer to the immediate advantage of the users. This is also particularly useful for the corrections of errors which might be identified by the developer or signalled by the users.

It is probably too early to foresee the future of electronic books which are just starting to spread, including in the field of education. The first examples of applications for educational material have just appeared. They can be found on the ipad in $(20,21)$ for the solar system or for the periodic chart of the elements and show the great potential of such devices. Some universities have already recognized this potential and distribute these new 
types of devices to their students. I am very optimistic and foresee a great future for this in the domain of education. It should be noted here that the expression 'electronic book' is an understatement. Not only do these devices show text documents, but they also contain very powerful CPUs to display animations and, most importantly, to simulate processes. Without doubt, we shall see in the near future the development of efficient tools for process simulation. I see here an excellent opportunity for crystallographers to transfer and adapt their applets and applications available on older platforms in order to be in better position to appeal to the new generation of students.

\section{Notes on contributor}

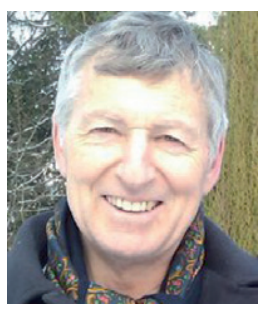

Gervais Chapuis received his $\mathrm{PhD}$ degree in Physics at the Swiss Federal School of Technology in Zurich (CH) (1971) and has completed Postdoctoral research at the Lawrence Berkeley Laboratory, Berkeley, CA (1972-1975). He was a Lecturer and later full Professor at the Department of Physics, University of Lausanne (1975-2003). He is a full Professor of Physics and Crystallography at the Swiss Federal School of Technology (EPFL) in Lausanne (since 2003). He is also a specialist in structural studies of solids by X-ray diffraction, structure-physical properties relations, theoretical and experimental studies of aperiodic crystals and in particular, incommensurate crystals and numerical modelling of aperiodic structures. He has developed new teaching tools for physics, chemistry and material sciences, information technology and communications tools for students, web-based teaching tools in the field of crystallography and structural sciences and crystallography teaching tools on mobile phones. He is the author of more than 250 scientific publications in refereed journals.

\section{References}

[1] Orlov, I.; Schoeni, N.; Chapuis, G. Crystallography on Mobile phones. J. Appl. Crystallogr. 2006, 39 (4), 595-597.

[2] Orlov, I.; Chapuis, G. Crystallography on iPhone [online]. http://escher.epfl.ch/iphone/ (accessed Mar 11, 2011).

[3] Schoeni, N.; Hardaker, W.; Escher, G. Web Sketch [online]. http://escher.epf1.ch/escher/ (accessed Mar 11, 2011).

[4] Orlov, I.; Chapuis, G. Crystallography on Mobile Phones [online]. http://escher.epfl.ch/mobile/ (accessed Mar 11, 2011).

[5] Weber, S. Java Applets [online]. http://jcrystal.com/steffenweber/java.html (accessed Mar 11, 2011)

[6] Schoeni, N.; Chapuis, G. Introduction to the Symmetry of Polyhedra [online]. http:// escher.epfl.ch/symmetry/ (accessed Mar 11, 2011).

[7] Schoeni, N.; Chapuis, G. The Reciprocal Lattice Calculator [online]. http://escher.epfl.ch/ rlattice/ (accessed Mar 11, 2011).

[8] Schoeni, N.; Chapuis, G. DiffractOgram [online]. http://escher.epfl.ch/diffractOgram/ (accessed Mar 11, 2011).

[9] Schoeni, N.; Chapuis, G. Fourier Transform [online]. http://escher.epfl.ch/fft/ (accessed Mar 11, 2011).

[10] Zalkin, A.; Templeton, D.H. The Crystal Structures of $\mathrm{YF}_{3}$ and Related Compounds. J. Am. Chem. Soc. 1953, 75 (10), 2453-2458.

[11] Cowtan, K. Structure Factor Applet [online]. http://www.ysbl.york.ac.uk/ cowtan/sfapplet/ bigframe_sf1.html (accessed Mar 11, 2011). 
[12] Proffen, Th.; Neder, R. Interactive Tutorial about Diffraction [online]. http://www.lks.physik. uni-erlangen.de/diffraction/teaching.html (accessed Mar 11, 2011).

[13] Oszlányi, G.; Sütö, A. Ab Initio Structure Solution by Charge Flipping. Acta Crystallogr. Section A 2004, 60, 134-141.

[14] Oszlányi, G.; Sütö, A. Ab Initio Structure Solution by Charge Flipping. ii. Use of Weak Reflections. Acta Crystallogr. Section A 2005, 61, 147-152.

[15] Schoeni, N.; Chapuis, G. CrystalOgraph [online]. http://escher.epfl.ch/crystalOgraph/ (accessed Mar 11, 2011).

[16] ICSD - Inorganic Crystal Structure Database [online]. http://www.fiz-karlsruhe.de/icsd.html (accessed Mar 11, 2011).

[17] Schoeni, N.; Chapuis, G. ReciprOgraph [online]. http://escher.epfl.ch/reciprOgraph/ (accessed Mar 11, 2011).

[18] Chapuis, G.; Schoeni, N. E-crystallography Course [online]. http://escher.epfl.ch/ eCrystallography/ (accessed Mar 11, 2011).

[19] Khan Academy [online]. http://khanacademy.org/index.html (accessed Mar 11, 2011).

[20] The Elements: A Visual Exploration [online]. http://appadvice.com/appnn/2010/03/deluxe-ipadapp-elements-visual-exploration/ (accessed Mar 11, 2011).

[21] Solar System [online]. http://touchpress.com/titles/solarsystem/ (accessed Mar 11, 2011).

\section{Subject Index}

Applets 190, 191, 198, 200-202

Arabic decorations 192

Bilbao crystallographic server 192

Charge flipping 197

CIF file 198, 199

Coherent scattering 196

Convolution theorem 196

Copyright 200

CrystalOgraph 198

Debye-Scherrer 194

Diffraction introduction to 196

DiffractOgram 193, 194

Diffuse scattering 196

Electron density 195-197

Electronic books 200, 201

Escher Web Sketch 191

Ewald sphere 193, 194

Exercises 189, 200, 201

Fixpoint operation 192

Fourier summation 196, 197

Fourier term 196

Fourier transform 188, 195-197

Fast 190, 195

Glide planes 191

ICSD database 198

iPad 201

iPhone 192

iTunes U 190, 201

IUCr 189, 200

Khan academy 201

Laue diagrams 194

Laue method 194 
Laue reflection 194

Laue transmission 194

Modular approach 201

Patterson function 195, 196

Phase problem 196-198

Polyhedra of point groups 192

Position general 189, 191, 192

Position special 189, 191, 192

Powder diagrams 194

Precession 194

Reciprocal lattice calculator 193

Reciprocal space 196, 197

ReciprOgraph 199

Rotation improper 191, 192

Rotation proper 191

Rotation screw 191

Space groups 191, 192

Stereographic projection 192, 193

Stoechiometry 192

Symmetry 189-193, 199, 200

Symmetry of polyhedra 192

Video recordings 190, 201

Weissenberg diagram 196

YouTube 190, 201 